\title{
Trabajo colaborativo docente: nuevas perspectivas para el desarrollo docente.
}

Teachers' collaborative work: new toward for teacher's development.

\section{Carolina Aparicio-Molina, Felipe Sepúlveda-López ${ }^{1}$}

RESUMEN: La educación chilena atraviesa por un proceso de cambios estructurales impulsados por nuevas reformas educacionales. La apropiada implementación del cambio educativo necesariamente ocurre a través de los principales actores encargados de la implementación de las reformas: Los docentes. Las investigaciones sobre el tema coinciden en que la implementación de reformas introduce elementos de tensión entre el profesorado, situación que se suma a los desafios que enfrentan los establecimientos educacionales situados en comunidades educativas con altos indices de vulnerabilidad socioeconómica. Esta investigación cualitativa, basada en un estudio de caso, explora el discurso de los docentes en ejercicio con respecto a su experiencia de trabajo colaborativo con sus pares contemplando los factores facilitadores y obstaculizadores del mismo. La información obtenida permite interpretar que la colaboración entre pares y su implicancia a lo largo del desarrollo profesional de los docentes se vincula a las distintas etapas vitales y de inserción laboral.

PALABRAS CLAVE: Profesionales de la Educación-Vida activa-Análisis cualitativo

ABSTRACT: Chilean education is undergoing through a process of structural change driven by new educational reforms. Proper implementation of educational change necessarily involves the main actors responsible for reforms implementation: Teachers. Researchers agree that reforms implementation introduces elements of stress among teachers. This situation adds to the challenges faced by schools located in communities with high rates of social vulnerability. This qualitative

${ }^{1}$ Universidad Católica de la Santísima Concepción, Chile.

Email: caparicio@ucsc.cl

Recibido: 28/08/2018 Aceptado: 17/04/2019

Doi: $10.18004 /$ riics.2019.junio.119-133

$(c)$ EY

ISSN (Impresa) 2225-5117. ISSN (En Línea) 2226-4000.

Rev. Int. Investig. Cienc. Soc. Vol. 15 n $^{\circ} 1$, junio, 2019.pág. 119-133 
Aparicio-Molina, C. et.; Trabajo colaborativo docente: nuevas perspectivas para el desarrollo...

research, based on a case study, explores the practicing teacher's discourse regarding their obstacles and facilitators factors based on their experiences with collaborative work with peers. The data obtained shows that collaboration between peers is associated with teachers' professional development and their working experiences as teachers.

KEYWORDS: Educational population - Working life - Qualitative analysis

\section{INTRODUCCIÓN}

La experiencia de aprender abre al ser humano al mundo, esto ocurre una y otra vez en la vida y especialmente en la escuela, sin embargo, dicha experiencia no es exclusiva de los estudiantes, sino que, para un adecuado desarrollo profesional, esta experiencia también debe ser vivida por los adultos que acompañan los procesos de aprendizaje: los profesores. De esta manera, el trabajo docente permite que quien lo realice experimente constantemente la magia de la conexión con un nuevo saber, ya sea con los estudiantes, los profesores, los padres o una técnica. En definitiva el aprendizaje es inseparable de la acción, como lo ha explicado Senge y colaboradores (Senge et al., 2012: 43), aspecto que especialmente en la trayectoria docente, configura el espacio de desarrollo de la profesión docente en la experiencia (Schön, 1998; Tardif, 2004).

Esta comprensión de lo que debe ocurrir en una escuela para el desarrollo profesional de los docentes, se expresa conjuntamente con el apremio por impulsar mejoras educativas a nivel internacional y nacional (Hargreaves y Fullan, 2014). Especialmente en Chile, en los últimos años se ha observado una constante reestructuración en los componentes del sistema educativo, elemento que, como expresara Fullan (2012), en materia de profesorado ha destacado por promover cambios al ser y quehacer docente en la cotidianeidad y burocracia del trabajo del profesor a través de la medición de su desempeño. Estos cambios se han traducido en la promulgación e implementación del Sistema Nacional de Evaluación Docente (SNED), el Programa de Integración Educativa y la incorporación de sistemas de codocencia, además de la entrada en vigencia de la Carrera Profesional Docente. 
Todos estos aspectos buscan promover un desarrollo profesional acorde a la mejora de la calidad de la enseñanza desde la formación inicial hasta la docencia en el sistema educativo nacional, lo cual espera revertir el proceso de desvalorización de la profesión docente en Chile (Cornejo, 2009; Hochschild, Díaz, Walker, Schiappacasse, y Medeiros, 2014) y fortalecer la educación pública (Valenzuela \& Montecinos, 2017).

Estos cambios en la estructura del trabajo docente, para autores como Fullan (2012) son uno de los elementos que detonarian un clima de tensiones ante la incertidumbre de lo desconocido y el ejercicio de un rol en el cual no se es tomado en cuenta como profesional. Esto en tanto en varios aspectos como por ejemplo el salarial, la jornada laboral y las horas de docencia, los docentes de Chile declaran no sentirse escuchados (Acuña, 2015; Cornejo, 2009). Sumado a lo anterior, el exceso de cambios en el quehacer, principalmente administrativo de los docentes se ha indicado como un factor que limitaria el éxito de las reformas. Especialmente al predominar la poca conexión entre las organizaciones que mandatan una reforma, la cultura de las instituciones y las personas implicadas en su aplicación (Hargreaves y Fullan, 2014; Marzano, Waters \& McNulty, 2005; Spillane, 2005).

En consideración de que un rasgo fundamental de la profesión docente es que su desarrollo ocurre junto a otros. Es importante tomar en cuenta que el saber del educador lleva consigo las marcas de su trabajo, es decir se construye en el encuentro con otros a través de la docencia. Este aspecto es esencial en el desarrollo profesional de los docentes, el cual estaría marcado por experiencias individuales del ejercicio junto a otros (Tardif, 2004: 15).

En consecuencia, es importante comprender que el trabajo colaborativo proviene de la visión de una cultura laboral en la cual se comparten fines y se opera interdependientemente para lograr los objetivos comunes. De esta manera la colaboración es un medio para alcanzar fines colectivos que permite el aprendizaje de actitudes, hábitos, creencias y saberes prácticos. Las relaciones colaborativas 
son así una vía a través de la cual los docentes salen de la denominada "ecología de la caja de huevos" (Goldenberg, 2003: 10) en la cual no se establecerían relaciones con los pares, para fortalecer los lazos personales ni mejorar el aprendizaje de los estudiantes.

Otro aspecto relevante del trabajo colaborativo dice relación con la idea de que quienes ejecutan este tipo de trabajos están en igualdad de condiciones, es decir la interdependencia de conocimientos y habilidades supone que no hay un único experto al cual seguir, sino que todos quienes se encuentran en el grupo de trabajo deben generar aportes para alcanzar el fin propuesto. De esta manera se evidencia que el trabajo que se emprende junto a otros genera favorables aprendizajes para las personas involucradas, lo que ha sido identificado en el campo educativo como un referente de escuela eficaz, además de un evidente indicador de inclusión escolar como han indicado Ainscow \& Sandill (2010).

Lo descrito evidencia frente a las particularidades del trabajo docente, en un contexto de reformas es clave comprender el contexto donde se plantean las innovaciones. En consecuencia la investigación ha indicado que, reconociendo el valor del docente para la mejora de los aprendizajes, más que el cambio individual, una organización educativa requiere de un cambio en la cultura del trabajo colaborativo para promover mejoras sostenibles en los aprendizajes de los estudiantes y conjuntamente facilitar el desarrollo profesional de los docentes, como lo han analizado reconocidos investigadores entre los que destacan Ainscow, Dyson, Goldrick, \& West, 2012; Darling-Hammond, Holtzman, Gatlin, \& Vasquez Heilig, 2005; Hargreaves y Fullan, 2014; Harris, 2012.

La evidencia internacional presentada es sin duda un espejo de la realidad chilena, en tanto Ávalos (2013) y un equipo de investigadores, indicaron la necesidad de aumentar el tiempo para organizar el trabajo del profesor, dándole especial importancia a generar espacios para trabajar colaborativamente entre docentes a fin de mejorar sus prácticas a través de la ayuda entre pares. Esta recomendación se corresponde con una publicación posterior de López \& Gallegos (2014), quienes relacionaron el trabajo colaborativo impulsado por los directivos de 
centros educativos, con la mejora en la satisfacción de los docentes con su desempeño en el aula y en la institución en general. Así como también lo observó Flores (2014) a través del análisis de los procesos de inducción a docentes nóveles a través de procesos informales realizados con otros profesores. Este proceso en el contexto nacional ha generado conflictos, porque si bien se reconoce la importancia del profesor para el éxito escolar y la mejora de la calidad de los procesos de aprendizaje, las reformas docentes no han revisado las diferencias entre la profesionalización del rol según los propios docentes versus la propuesta de avance solamente en incentivos económicos, como se ha dicho plantea la carrera profesional en Chile (Acuña Ruz, 2015).

Frente a este panorama nacional e internacional se hace necesario revisar en la práctica cómo el trabajo colaborativo hace apalancamiento a favor de las mejoras educativas y del propio desarrollo profesional docente para así converger en qué aspectos de las reformas educativas chilenas se podría potenciar el trabajo entre pares, preguntas que orientaron este trabajo de investigación.

En consecuencia, las perspectivas subjetivas respecto al trabajo colaborativo entre docentes se abordarán a partir de los discursos de docentes en ejercicio que plantearon su experiencia con pares de un mismo establecimiento educacional.

\section{METODOLOGÍA}

La investigación presenta un enfoque cualitativo y correspondió a un estudio de caso (Stake, 1998) en un centro de educación secundaria obligatoria de carácter técnico y científico humanista de la costera ciudad de Talcahuano, Región del Bíobío. El establecimiento educativo se caracteriza por tener un alto porcentaje de jóvenes con vulnerabilidad socioeconómica entre el 67 y 100\% anualmente, es decir atender un grupo con un perfil socioeconómico bajo, como se establece en su Informe de resultados en la prueba nacional SIMCE el Ministerio de Educación de Chile. 
El profesorado del liceo se divide entre aquellos que realizan docencia en asignaturas científico humanista y quienes realizan cursos técnicos como Atención de Adultos Mayores; Atención de Párvulos; Elaboración Industrial de Alimentos y Servicios de Alimentación Colectiva. El establecimiento se encuentra acogido a la Ley $\mathrm{n}^{\circ} 20.248$, Subvención Escolar Preferencial (SEP) y posee un equipo a cargo de implementar el Programa de Integración Escolar (PIE), para acoger las Necesidades Educativas Especiales de los jóvenes del liceo.

La información obtenida acerca del trabajo colaborativo se recopiló por medio de entrevistas en profundidad a docentes activos, con el objeto de develar a través del discurso individual las principales características del trabajo colaborativo que desarrollaban en su establecimiento, asi como los factores que facilitarian y limitarian el trabajo entre pares. El periodo de investigación correspondió a un semestre escolar. El guion de entrevistas fue validado temáticamente según áreas de desarrollo del trabajo colaborativo.

En total, se realizaron nueve entrevistas en profundidad a docentes que accedieron voluntariamente a participar de la investigación. Los profesores en ejercicio además cumplian con las características de realizar docencia en Enseñanza Media y estar contratados por más de media jornada en el establecimiento educacional. Los docentes entrevistados fueron informados del contenido de la entrevista, accedieron a ser grabados y a que la información sea utilizada con fines investigativos a través de un consentimiento informado.

Los resultados fueron analizados a partir del software ATLAS.ti, en el cual se generaron códigos a partir de los datos obtenidos, siendo un total de 146 códigos los emergidos de las entrevistas. Posteriormente, en función de la emergencia de reiteraciones y sentidos comunes entre los códigos se levantaron 9 categorias o dimensiones de análisis (Trinidad, Carrero y Soriano, 2006).

\section{RESULTADOS}

Los códigos emergidos tras la saturación de los datos, permitió establecer 
relaciones entre los distintos tópicos interpretados, de modo de conectar los diversos aspectos que involucrarian el desarrollo de trabajo colaborativo en un contexto específico, como el centro educativo en cuestión. De esta manera las categorias emanadas del discurso individual de cada docente lograron aunar visiones compartidas respecto al trabajo colaborativo. Como se observa en la siguiente red semántica elaborada a partir de las categorías:

Figura 1: Red semántica generada a partir del análisis de las entrevistas por medio de software de análisis Atlas Ti

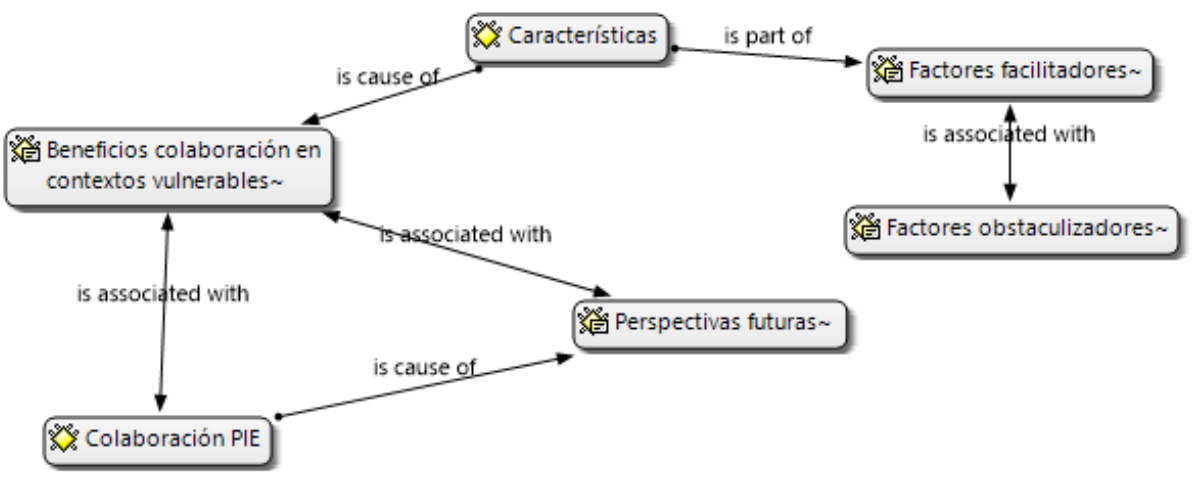

Fuente: Elaboración propia.

Se evidenció que los profesores identificaban especialmente la necesidad de apoyarse entre pares y buscar estrategias comunes para desempeñarse en contextos que ellos consideraban dificiles por la vulnerabilidad social de los estudiantes, aspecto que generalmente describian como distinto a lo conocido y de alta exigencia emocional. Esta característica, explicada por la alta segregación por origen socioeconómico que persiste en la realidad educativa chilena (OCDE, 2004; Valenzuela, Bellei y De Los Ríos, 2011) es una condición que, desde la informalidad les permite a los profesores conectarse entre ellos y ayudarse mutuamente, especialmente a los profesores nóveles, como había indicado Flores (2014). 
Por ello una característica del trabajo colaborativo es lo beneficioso de esta experiencia en contextos vulnerables y cómo los docentes estimarían necesario que a futuro se potencie este aporte particular del trabajo colaborativo. Además, estos elementos los asociaron con las evidencias que habian obtenido a través de los procesos de colaboración formal en el marco de los programas de inclusión (PIE). Este programa, entre otras cosas, les habría permitido alcanzar un mejor dominio del grupo curso al contar con un apoyo permanente en el aula, especialmente para el necesario control de la disciplina, tanto porque los jóvenes no aprendian a un mismo ritmo, como por la falta de hábitos académicos en los estudiantes. A causa de esta experiencia y la interpretación dada por los docentes, se identificó como perspectiva futura de la colaboración, el trabajo conjunto en el aula, es decir la codocencia (Mineduc, 2012).

Sin duda la co-docencia implicaria una serie de desafios asociados a los factores facilitadores y obstaculizadores identificados por los docentes, en tanto el factor tiempo fue considerado altamente relevante para diseñar e implementar una co-docencia que permitiera a ambos profesores trabajar adecuadamente. A su vez las características de los educadores diferenciales, como la creatividad y búsqueda del aprendizaje de todos los estudiantes, fue reconocida como un elemento a favor del desarrollo de esta metodología de docencia, como se observa en la siguiente cita:

"me ayuda con otros alumnos que no están en su grupo, ella también les ayuda; compartimos el trabajo en la sala de clases y eso es mucho más eficiente" (E5:15-16)

Por otra parte, los datos obtenidos permitieron interpretar que la colaboración entre pares y su implicancia a lo largo del desarrollo profesional de los docentes se vincularía igualmente con las distintas etapas vitales y de inserción laboral. En tanto los docentes más jóvenes atribuyeron principalmente los beneficios de la colaboración a la posibilidad de aprender a trabajar con los estudiantes adecuándose a su realidad socioeconómica, aspecto que no había sido considerado en su formación de pregrado, como han enfatizado investigadores 
nacionales (Ferrrada, Villena y Turra, 2015). De esta forma, la iniciación de una trayectoria profesional demandaría un proceso de inducción, en los saberes propios de la práctica docente pero también en la realidad sociocultural de los jóvenes y sus familias (Flores, 2014), aspectos que con acierto han sido considerados en la Política Nacional Docente en Chile.

\section{DISCUSIÓN}

Los reconocidos estudios de Eddy (1971) y Lortie (1975) (en Tardif, 2004) habian identificado etapas en la trayectoria docente las cuales estaban delimitadas por su integración en la comunidad de profesores del establecimiento en el cual se desempeñaban. Donde Eddy (1971) identificó tres fases en la iniciación en la carrera docente: la primera correspondiente a la transición del estudiante al docente, en la cual debía seguir reglas para ser eficiente en la contención de los alumnos; la segunda correspondiente a la aceptación de la jerarquía docente y la importancia de cumplir con las reglas informales de la sala de profesores; la tercera era finalmente el descubrimiento de los alumnos reales, los cuales no necesariamente estaban deseosos de aprender o ser sensibles a las recompensas y castigos del docente.

Por su parte Lortie (1975) identificó una fase de exploración (de uno a tres años) donde se comenzaban a asumir las funciones de la enseñanza mediante ensayo y error, para ser aceptado y validado. Etapa en la cual muchas veces los jóvenes abandonaban la docencia; y otra fase de estabilización y consolidación (tres a siete años), en la cual el profesor era reconocido por la institución, poseía más confianza en sí mismo y dominaba más aspectos del trabajo.

Ambas investigaciones han sido fundamentales en la comprensión del desarrollo profesional docente, sin embargo, se hace necesario considerar en la inserción del profesor a las aulas, las características estructurales de la educación, como está demarcado especialmente en Chile. En consecuencia, la segregación 
educativa y las consecuencias que tiene ésta en el desarrollo profesional docente convocan a revisar una primera etapa en el desarrollo profesional docente de "ajuste" entre la realidad educativa y los propios saberes culturales, especialmente conectado con la cultura de los propios estudiantes.

Esta perspectiva se justifica con la información registrada en la comuna de Talcahuano, en tanto entre la categoría de factores obstaculizadores y facilitadores, se evidenció la fuerza de las relaciones interpersonales para promover o limitar el trabajo colaborativo entre pares. Esto en tanto, pese a mantener fines comunes, los docentes reconocieron la problemática situación de trabajar con pares cuando no existía afinidad ni mismos grados de compromiso. Situación ante la cual los profesores jóvenes reconocieron la necesidad de contar con un mayor apoyo de los pares para resolver problemas de aula y de desarrollo curricular en un contexto sociocultural vulnerable que suponía crisis de disciplina y dificultades para concretar el proceso de enseñanza- aprendizaje como lo tenían previamente definido.

Así, el diseño de estructuras organizacionales que promuevan una cultura de colaboración, basada en el mejoramiento colectivo y la construcción de aprendizajes compartidos se hace relevante especialmente cuando se imparte educación en contextos de vulnerabilidad social. La promoción de una cultura de colaboración entre pares debe ser un proceso facilitado por los equipos directivos del establecimiento. En este sentido, no basta sólo con declarar la necesidad del mejorar el trabajo en equipo, se deben crear espacios reales dentro de la rutina escolar que entreguen tiempo para la reflexión colectiva y el análisis de las prácticas pedagógicas efectivas dentro del cada equipo docente.

Todo lo anterior se relaciona con lo previamente explicado por Tardif, en tanto los profesores a lo largo de su carrera profesional, construyen un saber que se caracteriza por ser social. Social en el sentido de que emerge en un espacio colectivo y se legitima junto a los otros profesores a través de reglas determinadas por un sistema. En consecuencia, el saber de los maestros estaria en constante transacción entre lo que son individualmente es decir sus emociones, historia, 
cognición, expectativas y lo que hacen en el trabajo escolar junto a sus pares y estudiantes (Tardif, 2004: 11-14).

Los antecedentes revisados invitan a la reflexión sobre el trabajo colaborativo en Chile, reflexión que debe ser situada desde la perspectiva colectiva del ser y ejercer como docente. Aspecto que, en este país, se relacionaría estrechamente con las características socioculturales del entorno en el cual se desempeñaría el docente, promoviendo o limitando las frustraciones del profesor, especialmente del novel, respecto a sus capacidades y desarrollo profesional.

En consecuencia, a partir de los elementos revisados se pone en evidencia la necesidad de abordar la mejora educativa desde una perspectiva integral que comprenda un necesario ajuste y mejora a la formación continua de los docentes y a la evaluación que se realiza de su labor, en tanto existen demandas por mejorar la co docencia y establecer lazos de cooperación entre pares para impactar positivamente en los aprendizajes de los estudiantes.

Junto con lo anterior, se hace necesario conocer y reconocer la cultura docente y el contexto donde se significan las prácticas pedagógicas. Por ello, frente a la implementación de una política de mentorías para los profesores nóveles, se hace especialmente relevante que esta politica considere las características contextuales de las aulas chilenas. De modo que se integren las principales dificultades de los contextos vulnerables como un tema real y empírico, permitiendo al docente ajustar efectivamente sus expectativas a la realidad para que la innovación y mejoras que proponga a su propio ejercicio docente se ancle en un escenario concreto. De esta manera quienes ejerzan de mentores no podrian ser si no personas experimentadas en el mismo contexto educativo al que ingresa el joven profesor, pero que además ha podido sobresalir a las dificultades del contexto. Estos elementos sin duda lo impulsarán a motivar e invitar al profesor a disfrutar del proceso de inserción laboral y principalmente estimular su desarrollo profesional en las distintas etapas de su vida como docente.

Por último, el reconocimiento de las tensiones existentes entre el sentido 
del trabajo colaborativo en contextos vulnerables con la dificultad de desempeñarse en estos contextos y especialmente la limitante del desencuentro generacional. Son elementos que revelan la importancia de incorporar un sentido de capacidades en los procesos de implementación de las innovaciones que se desarrollan, como elemento que dé sostenibilidad a los cambios ya que, aunque categórico, los estudios analizados por Fullan (2012) indican que "el cambio siempre fracasará si no encontramos un modo de crear infraestructuras y procesos que involucren al profesorado en el desarrollo de nuevas concepciones" (2012:68).

Es así como, en esta parte del desarrollo de la carrera docente en Chile se podría encontrar el espacio para permitir a los docentes que se apropien de su quehacer y potencien las mejoras de sus establecimientos desarrollando sus propias capacidades. La investigación educativa busca de esta manera colaborar en los procesos de implementación.

\section{REFERENCIAS}

Acuña Ruz, F. (2015). Incentivos al trabajo profesional docente y su relación con las políticas de evaluación e incentivo económico individual. Estudios Pedagógicos (Valdivia), 41(1), 7-26. http://doi.org/10.4067/S071807052015000100001

Ainscow, M., Dyson, A., Goldrick, S., \& West, M. (2012). Making schools effective for all: rethinking the task. School Leadership \& Management, 32(3), 197213. http://doi.org/10.1080/13632434.2012.669648

Ainscow, M., \& Sandill, A. (2010). Developing inclusive education systems: the role of organizational cultures and leadership. International Journal of Inclusive Education, 14(4), 401-416. http://doi.org/10.1080/1360311080250493

Ávalos, B., (editora). (2013). Héroes o villanos. La profesión docente en Chile. Santiago: Editorial Universitaria.

Cornejo, R. (2009) Condiciones de Trabajo y Bienestar/Malestar Docente en 
Profesores de Enseñanza Media de Santiago de Chile. Educ. Soc., Campinas, vol 30, n. 107, p. 409-426, maio/ago.

Darling-Hammond, L., Holtzman, D. J., Gatlin, S. J., \& Vasquez Heilig, J. (2005). Does Teacher Preparation Matter? Evidence about Teacher Certification, Teach for America, and Teacher Effectiveness. Education Policy Analysis Archives, 13, 42. http://doi.org/10.14507/epaa.v13n42.2005

Ferrrada, D., Villena, A., y Turra, O. (2015). Transformar la formación. Las voces del profesorado. Santiago: Universidad Católica de la Santísima ConcepciónRIl Editores.

Flores, C. (2014) Inducción de profesores novatos en Chile: un estudio de caso. Pensamiento Educativo. Revista de Investigación Educacional Latinoamericana. 51(2), 41-55

Fullan, M. (2012). Los nuevos significados del cambio en la educación. Barcelona: Octaedro.

Goldenberg, C. (2003). Setting for School Improvement. International Jounal of Disability, Development and Education, 50(1), 7-16. http://doi.org/10.1080/1034912032000053304

Hardre, P. L., Ling, C., Shehab, R. L., Nanny, M. A., Nollert, M. U., Refai, H., ... Wollega, E. D. (2013). Teachers in an Interdisciplinary Learning Community: Engaging, Integrating, and Strengthening K-12 Education. Journal of Teacher Education, 64(5), 409-425. http://doi.org/10.1177/0022487113496640

Harris, A. (2012). Liderazgo y Desarrollo de capacidades en la escuela. Santiago: Fundación Chile- CAP.

Hargreaves, A. y Fullan, M. (2014). Capital Profesional. Madrid: Morata.

Hochschild, Hernán, Díaz, Facundo, Walker, Joaquín, Schiappacasse, Jorge y 
Medeiros, María Paz. (2014). El Plan Maestro, diálogos para la profesión docente. Calidad en la educación, (41), 121-135. Recuperado en 09 de marzo de 2016, de http://www.scielo.cl/scielo.php?script=sci_arttext\&pid=S0718$45652014000200005 \& \operatorname{lng}=$ es $\&$ tlng $=$ es. $10.4067 /$ S071845652014000200005 .

López A, P. y Gallegos A, V. (2014). Prácticas de liderazgo y el rol mediador de la eficacia colectiva en la satisfacción laboral de los docentes. Estudios Pedagógicos (Valdivia), 40(1), 163-178. http://doi.org/10.4067/S071807052014000100010

Marzano, R. J., Waters, T. \& McNulty, B. A. (2005). School Leadership That Works: From Research to Results (1st ed.). Alexandria,VA: ASCD.

Mineduc (2012). Decreto Supremo No 170/09. Orientaciones Técnicas para PIE. Trabajo Colaborativo y co- Docencia. Recuperado de http://portales.mineduc.cl/usuarios/edu.especial/doc/201209121910450.P PT_DS170_04_Trabajo_Colaborativo.pdf

OCDE (2004): Revisión de políticas nacionales en educación: Chile. París y Santiago: Ocde y Mineduc.

Senge, P., Cambron- McCabe, N., Lucas, T., Smith, B., Dutton, J., \& Kleiner, A. (2012). Schools that learn. New York: Crown Business.

Schön, D. (1998). El profesional reflexivo. Cómo piensan los profesionales cuando actúan. Barcelona: Paidós.

Spillane, J. P. (2005). Distributed Leadership. The Educational Forum, 69(2), 143150.

Stake, R. (1998). Investigación con estudios de caso. Madrid: Morata.

Tardif, M. (2004). Los saberes del docente y su desarrollo profesional ( $3^{\circ}$ ed.). Madrid: Narcea. 
Trinidad, A. Carrero, V. y Soriano, R. (2006), Teoria fundamentada "Grounded Theory”. La construcción de la teoría a través del análisis interpretacional, Cuaderno metodológico 37, CIS, Madrid.

Valenzuela, J., Bellei, C., y De los Ríos, D. (2011). Segregación escolar en Chile. Fin de ciclo: Cambios en la gobernanza del sistema educativo. Facultad de Educación, Pontificia Universidad Católica de Chile y Oficina Regional para América Latina y el Caribe UNESCO.

Valenzuela, J. P., \& Montecinos, C. (2017). Structural Reforms and Equity in Chilean Schools. http://doi.org/10.1093/ACREFORE/9780190264093.013.108 